\title{
Parents' Awareness of Dental Caries Prevention in Pediatric Patients
}

\author{
Sun Hye Jung ${ }^{1}$, Jin Young Gam, Ye Rim Park', Ye Lim Sin ${ }^{1}$, Sun Myung Lee ${ }^{1}$, So Hui Im", \\ Hyun Wook Jeong ${ }^{1}$, Young Eun Lee ${ }^{1}$ \\ ${ }^{1}$ Department of Dental Hygiene, Daegu Health College, Daegu, Republic of Korea
}

Objectives: Proper oral care in infancy and early childhood is the foundation for maintaining the habit of caring for teeth throughout life. If habits are not properly formed during infancy and early childhood, it can lead to bad results not only in dental caries but also in chronic periodontal disease in adulthood. Therefore, the role of parents is essential for children's oral health. The purpose of this study was to investigate the awareness level of dental caries prevention among parents with children from infancy to adolescence.

Methods: This study conducted an online survey on May 2021, targeting 119 parents of with children or parents of students residing in Daegu Metropolitan City. A structured self-administered questionnaire was used for a survey.

Results: As a result of this study, it was found that although $79 \%$ of parents were interested in their children's dental health, $64.7 \%$ of children did not actually use oral hygiene products. $66.4 \%$ of children usually enjoyed eating sweet food, and $74.8 \%$ of children did not brush their teeth immediately after eating sweet food. $61.3 \%$ of parents had never received education on brushing or how to use oral hygiene products about their children's oral care, and 55\% of parents regularly took their children to the dentist.

Conclusions: Summarizing the above results, most parents are interested in their children's dental health and have received education on brushing, while the use of oral hygiene products and the need for prevention of dental caries in pediatric patients are relatively low. Therefore, if the level of oral health knowledge is improved by implementing a dental caries prevention education program for parents, it will be of great help to prevent dental caries in children in the future.

Keywords: Pediatric patients, Dental caries prevention, Parents' awareness

Copyright (c) 2021. Korean Academy of Preventive Dentistry. All rights reserved.

This is an Open Access article distributed under the terms of the Creative Commons Attribution Non-Commercial License (http://creativecommons.org/licenses/ by-nc/4.0) which permits unrestricted non-commercial use, distribution, and reproduction in any medium, provided the original work is properly cited. 